\title{
Erratum to: "Low-Frequency Broadband Ultrasonic Transducers for Testing Articles that Are Manufactured of Large-Structure and Composite Materials. Part 2. Excitation of Low-Frequency Ultrasonic Wide-Band Signals" [Russian Journal of Nondestructive Testing 51, 407 (2015)]
}

\author{
A. I. Potapov ${ }^{a}$, V. E. Polyakov ${ }^{a}$, V. A. Syas'ko ${ }^{b}$, A. A. Popov ${ }^{b}$ and P. V. Kur'anova ${ }^{b}$ \\ ${ }^{a}$ National Mineral Resource University (University of Mines), 21 liniya 2, St. Petersburg, 199106 Russia \\ e-mail:apot@mail.ru,polykov1939@mail.ru \\ ${ }^{b}$ ZAO Konstanta, ul. Marshala Govorova 29, St. Petersburg \\ e-mail:9334343@rambler.ru \\ (Submitted October 27, 2015; accepted for publication October 27, 2015)
}

DOI: $10.1134 / \mathrm{S} 1061830915090119$

The list of authors and their affiliations should read as follows:

A. I. Potapov ${ }^{a}$, V. E. Polyakov ${ }^{a}$, V. A. Syas'ko ${ }^{b}$, A. A. Popov ${ }^{b}$, and P. V. Kur'anova ${ }^{b}$

${ }^{a}$ National Mineral Resource University (University of Mines), 21 liniya 2, St. Petersburg, 199106 Russia e-mail: apot@mail.ru,polykov1939@mail.ru

${ }^{b}$ ZAO Konstanta, ul. Marshala Govorova 29, St. Petersburg

e-mail:9334343@rambler.ru 